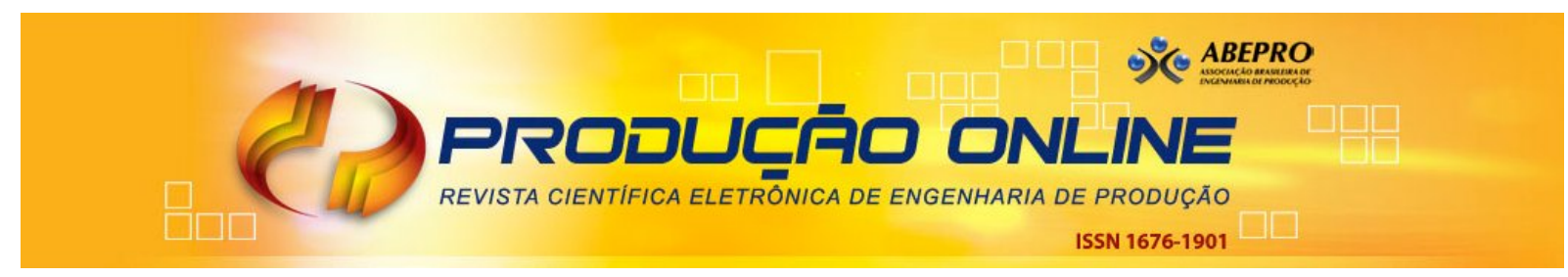

\title{
OTIMIZAÇÃO DOS CUSTOS DE FROTA URBANA COM USO DE MODELO COMBINADO DE LIFE CYCLE COST E SIMULAÇÃO DE MONTE CARLO
}

\section{URBAN FLEET COST OPTIMIZATION USING A LIFE CYCLE COST AND MONTE CARLO SIMULATION HYBRID MODEL}

\author{
Jorge Luiz Riechi* E-mail: riechi@utfpr.edu.br \\ Bernardo Tormos** Email: betormos@mot.upv.es \\ Marcos Vinicius Jacometo Hillebrand* E-mail: marcos.hillebrand@gmail.com \\ *Universidade Tecnológica Federal do Paraná (UTFPR), Curitiba, PR \\ ** Universitat Politècnica de València (UPV), València, Espanha
}

Resumo: Otimizar o valor do custo médio anual, de uma frota de ônibus, tem se tornado uma preocupação, cada vez maior, na gestão das empresas de transporte em todo mundo. Atualmente, existem várias ferramentas disponíveis para auxiliar as decisões gerenciais e, uma da mais utilizadas, é a análise do custo do ciclo de vida de um ativo, conhecida como "Life Cycle Cost". Caracterizada por executar uma análise determinística da situação, permite à gestão, avaliar o processo de substituição da frota, porém, mostra-se limitada, por não contemplar certas variações intrínsecas aos veículos e por, desconsiderar variáveis relacionadas às contingências de uso da frota. O objetivo principal deste trabalho é desenvolver um modelo combinado de apoio ao gerenciamento de ativos, baseado na associação entre a ferramenta Life Cycle Cost e o modelo matemático de Simulação de Monte Carlo, mediante a realização de uma análise estocástica, considerando tanto a idade, quanto a quilometragem média anual para substituição ótima de um veículo. O método utilizado foi aplicado em uma frota de transporte urbano brasileira, e os resultados indicam que o uso do modelo estocástico foi mais eficiente que a utilização de modelo único determinístico.

Palavras-chaves: Life Cycle Cost. Simulação de Monte-Carlo. Otimização. Substituição de Frota. Análise de Custo.

\begin{abstract}
The optimization of the annual average cost for a bus fleet had become an important issue for the managers of transport companies worldwide. Currently, there are several available tools to support managerial decision making. One of the most used techniques to analyze is the deterministic method named "Life Cycle Cost" which allows the user to assess the replacement moment. However, this method is limited because it does not consider all the possible intrinsic variations in the equipment or the possible modifications in the utilization level. This paper objective is to develop a tool to support asset's management through the combination of the Life Cycle Cost and the Monte Carlo Simulation approaches, which forms a stochastic analytical model that considers age, annual mileage for the optimal replacement fleet. For this paper's development, data obtained from a Brazilian company were employed. The results show that the use of this combined tool is more efficient that the deterministic model.
\end{abstract}

Keywords: Life Cycle Cost. Monte Carlo Simulation. Optimization. Replacement fleet. Cost Analysis. 


\section{INTRODUÇÃO}

Todas as atividades de manutenção de veículos têm como objetivo principal, preservar o estado dos mesmos o mais próximo possível com o apresentado quando novos e em condições ideais de uso, porém, com o passar dos anos e com o consequente desgaste, mantê-los torna-se cada vez mais oneroso, podendo chegar a uma situação de inviabilidade econômica. Em decorrência deste fato, deve existir um momento ótimo, o mais adequado para a substituição do veículo, que não coincide necessariamente com o tempo de vida e com a capacidade de desempenho das funções requeridas.

De acordo com o conhecimento atual em gestão de manutenção, é possível prolongar a vida do veículo de transporte com o aumento das técnicas de manutenabilidade, incluso os trabalhos de reparação geral em componentes chave como os elementos do sistema PowerTrain (DHILLON, 2010). Porém, para tal, duas perguntas precisam ser respondidas:

I. O período de viabilidade econômica do procedimento executado.

II. A eleição das prioridades da empresa de transporte, entre o aumento do período de vida de um ativo baseado no aumento de custos gerador de menor rentabilidade ou, a substituição do mesmo no período mais adequado.

Responder a estas questões e gerenciar os custos de operação e manutenção (O\&M) nunca é uma tarefa fácil. Os gestores responsáveis desta atividade devem controlar sempre os dados financeiros e técnicos, com o objetivo de maximizar a vida útil dos veículos, minimizar seus custos, conscientes de que o aspecto econômico sempre prevalece ao técnico, respeitando os aspectos de seguridade (DHILLON, 2010). Para as frotas de transporte urbano, considera-se outros fatores que influenciam o processo de substituição como: decisão estratégica de ampliação da capacidade da frota, obsolescência dos veículos, conveniência de substituição por redução de custos de aquisição influenciados por uma política governamental com isenção de impostos e/ou subsídios. Certamente, a melhor opção é a definição da política clara e sustentada cientificamente de renovação em função da quilometragem percorrida e da idade do veículo, desenvolvidas pela análise de custos. Outros fatores, como: aspecto e capacidade financeira da 
empresa, dimensão da frota, estrutura física, custos administrativos gerais, demanda dos serviços, podem também influenciar na tomada de decisão de substituição da frota. Porém, para adotar também estas considerações, seria necessário outro tipo de abordagem, que não contemplado neste estudo.

Alguns autores como Feldens et al. (2010), Dario et al. (2014), e Costa et al. (2014) sugerem que no processo de decisão, as empresas devam buscar o uso eficiente dos ativos fixos vinculado a uma estruturada política de avaliação e substituição da frota, considerando-se também a análise do custo de manutenção em relação à disponibilidade do ativo e o lucro da empresa.

Feng e Figliozzi (2012) apontam que se por um lado, a substituição de frota de veículos antigos por novos, pode reduzir os custos de manutenção, uma vez que, quanto mais antigo for o veículo maior seu custo, por outro lado, a substituição de frota aumenta significativamente os custos de capital. Esta questão, tornar-se mais complexa quando as decisões de substituição são feitas em grandes frotas e quando, o orçamento e as restrições de demanda são considerados. No entanto, os estudos demonstram a necessidade da identificação precisa do momento ideal de substituição da frota para a minimização do custo líquido ao longo da vida útil do veículo.

Estudos de Emblemsvag (2003) e Dhillon (2010), indicam que a ferramenta de análise do custo do ciclo de vida $\mathbf{L} C$, é cada vez mais utilizada para apoiar a tomada de decisões que direta ou indiretamente, se relacionam com equipamentos e/ou sistemas de engenharia e supera em muitos casos as deficiências da análise contábil simples, na gestão dos ativos das empresas. A questão essencial, não é a técnica, mas sim a econômica, que apresenta como objetivo principal maximizar o retorno sobre o investimento em ativos, obtendo um melhor desempenho e um menor custo de operação e manutenção. Neste contexto, há vários trabalhos que utilizam esta ferramenta, como mostrado nos estudos de Feldens et al. (2010), Zambujal e Duque (2011), Feng e Figliozzi (2012) e Mishra et al. (2013).

No entanto, a ferramenta LCC por ser uma abordagem determinística, apresenta algumas limitações ao analisar cenários em frotas de veículos, sendo necessário sua combinação com um modelo matemático que permita uma análise estocástica. Este modelo combinado, permite a análise mais ampla e confiável das 
variáveis que interferem direta e indiretamente no desempenho da frota e consequentemente da empresa (FAN, MACHEMEHL, GEMAR, \& BROWN, 2014) (EMBLEMSVAG, 2001).

Segundo Silveira et al. (2014), há vários modelos matemáticos com simulação para resolução de problemas estocásticos, disponíveis na literatura, destacando-se a Simulação de Monte Carlo (SMC), baseada na geração de números aleatórios e probabilidade de ocorrência de valores associados ao fenômeno estudado em análise. Dentre as vantagens da SMC, destaca-se que os dados de entrada podem apresentar qualquer tipo de distribuição, podendo analisar cenários de forma ágil (alterando somente dados de entrada) e, devido a não existência de um algoritmo único para SMC, pode-se ajustar o procedimento de simulação da forma mais oportuna à situação. Associado a esta premissa, Pamplona e Silva (2005) e Saraiva Júnior et al. (2010), entendem a SMC como um método numérico estocástico universal para a solução de problemas matemáticos, propondo sua utilização ao gerenciamento de riscos. Para Leal e Oliveira (2011) e Ritter et al. (2014), a SMC vem sendo aplicada com sucesso em gerenciamento de projetos, permitindo melhor análise de custos.

O presente estudo demonstra o modelo combinado de gerenciamento de frota, baseado na ferramenta de Análise do Custo do Ciclo de Vida LCC e no modelo matemático de Simulação de Monte Carlo (SMC). Quanto maior e melhor for a disponibilidade e confiabilidade dos dados, maior será o sucesso do modelo. Os dados coletados pertencem a uma frota de transporte urbano brasileira Empresa Z, da cidade de Curitiba-Paraná.

\section{METODOLOGIA E DESENVOLVIMENTO}

O fluxograma apresentado na figura 1 representa em síntese 0 desenvolvimento metodológico deste trabalho, demonstrando os procedimentos.

$\mathrm{Na}$ primeira etapa do trabalho foram coletados os dados da Empresa $Z$ para definir a extensão das informações e o perímetro das análises. A curva dos custos de manutenção e operação foi definida em função da idade do veículo e da quilometragem desenvolvida pelo mesmo. 
Figura 1 - Fluxograma da Sequência do desenvolvimento Metodológico do trabalho

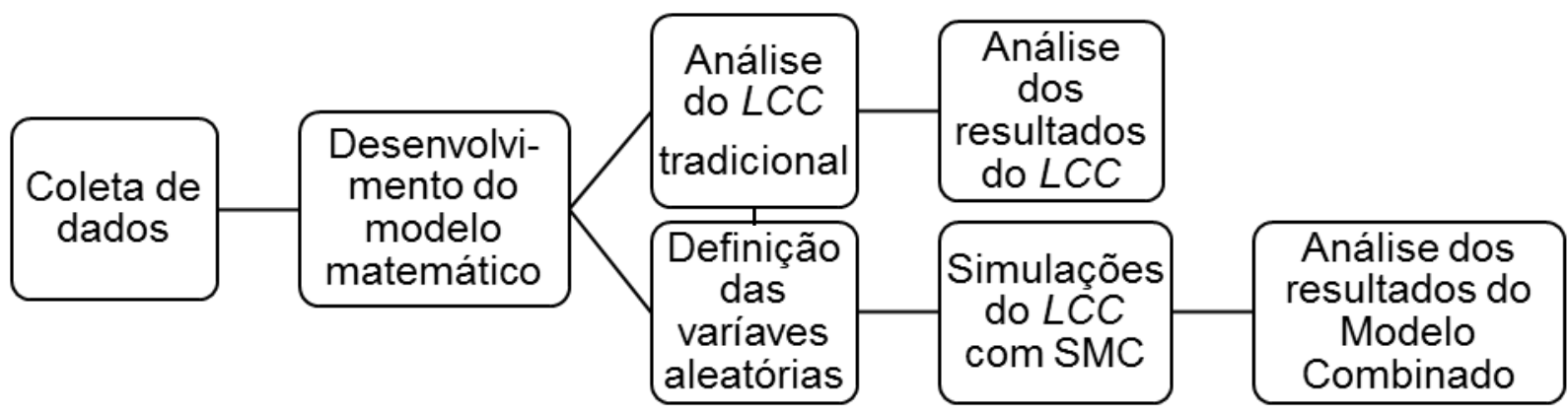

Posteriormente, com a base de dados analisada, foi desenvolvido o modelo matemático representativo do fluxo de caixa de um ativo, conforme considerado pelo LCC. Deste modelo matemático, foi criado o problema de otimização proposto, no qual buscou-se encontrar o ponto de mínimo valor do custo médio anual, o qual coincide com o ponto ótimo de substituição de um ativo, sob o aspecto econômico. Para tal, foi desenvolvida a função do custo de operação e manutenção de um veículo em função da sua idade e da sua utilização (quilometragem média anual). Nesta mesma fase, também foi explanado sobre o modelo de depreciação do ativo utilizado neste trabalho.

Com o modelo matemático tradicional definido, o método foi realizado em duas etapas. Inicialmente, foi realizado uma análise do ponto ótimo de troca para um ônibus através da ferramenta LCC. Em seguida, foi adicionado ao modelo matemático tradicional, o conceito de variáveis aleatórias, para que o mesmo, pudesse ser resolvido através da abordagem estocástica com a Simulação de Monte Carlo, possibilitando a análise do impacto da variabilidade no resultado final. A Simulação de Monte Carlo foi realizada para 10.000 cenários diferentes. O número de cenários definido mostrou-se adequado, uma vez que não foram encontradas diferenças significativas em análises comparativas com amostras maiores, em contraponto simulações com tamanhos de amostra de ordem inferior não são recomendados, pois podem representar a totalidade das combinações possíveis de variáveis aleatórias. Após a análise dos modelos de LCC tradicional e LCC com Simulação de Monte Carlo, foi desenvolvido um estudo comparativo entre os resultados dos dois modelos. 


\subsection{Análise da Base de Dados}

A amostra inicial foi composta por uma frota de 300 ônibus de diversas marcas (Volvo, Mercedes Bens, Scania) e modelos advindos de vários encarroçadores destinados ao transporte urbano de passageiros. Mediante a aplicação dos critérios de inclusão: a) características construtivas similares, fabricante, encarroçador, combustível (diesel), b) condições similares de serviço e quilometragem média anual, a amostra final foi constituída por 33 veículos. O banco de dados analisado foi seccionado em períodos anuais, entre os anos de 2011 a 2015, totalizando um período total de cinco anos de análise. Para efeito de cálculo, a idade considerada do veículo foi a do final do período analisado.

A figura 2 descreve a dispersão média das quilometragens anuais da amostra. Em função desta, foi definido a quilometragem anual média de $80.620 \mathrm{Km}$ /ano como valor base para análise do $\angle C C$, dado representativo do uso atual dos veículos. Para a análise do modelo combinado entre LCC e SMC, a quilometragem média anual considerada, foi fixada entre os intervalos de $40.000 \mathrm{Km}$ /ano e $100.000 \mathrm{Km} /$ ano, uma vez que, este intervalo representa $80 \%$ dos dados disponibilizados, garantindo melhor desempenho do modelo matemático.

Figura 2 - Descrição da quilometragem anual da frota analisada

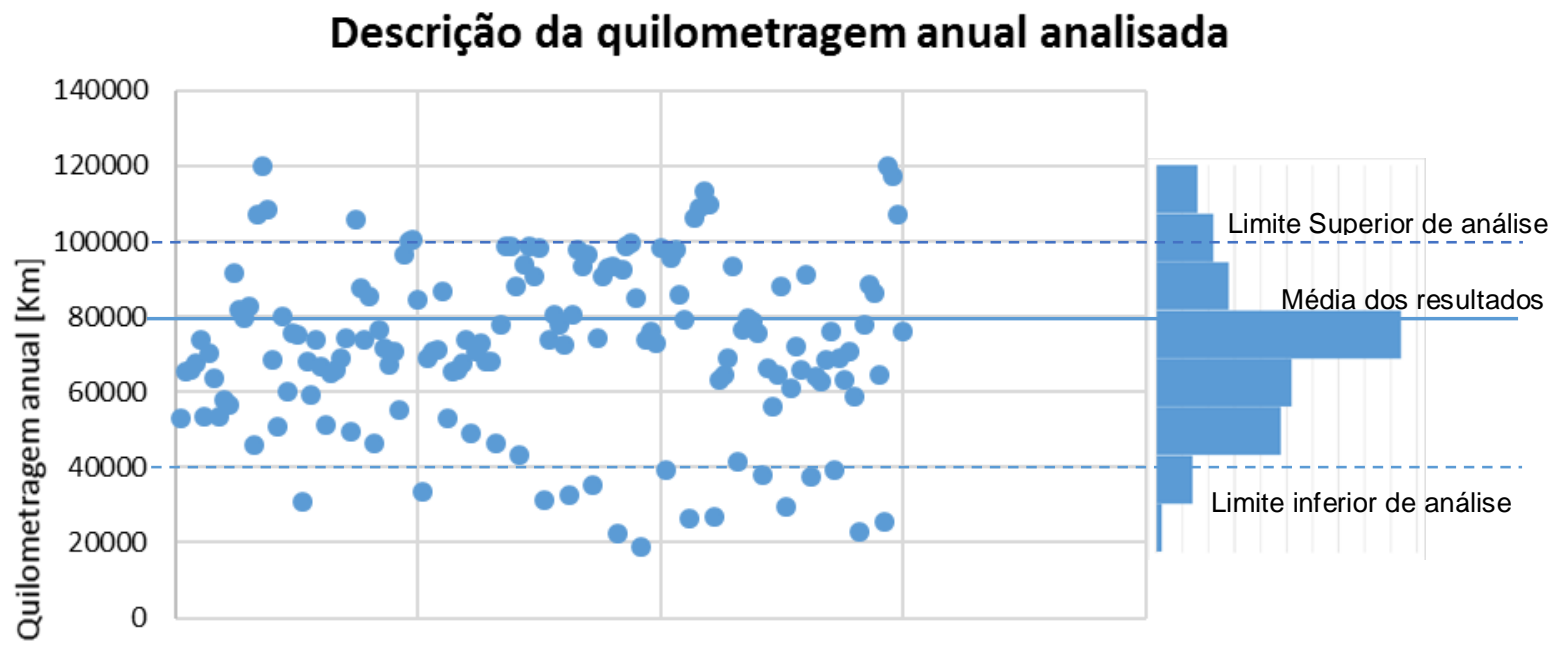

Com relação ao cálculo dos custos, algumas premissas foram consideradas durante o desenvolvimento, seguem descritas abaixo: 
1. O consumo médio de combustível foi considerado constante durante toda vida útil do veículo, pois mesmo sabendo que, na prática há uma pequena variação em função das condições do uso e uma leve tendência ao aumento do consumo com o envelhecimento do veículo, as mesmas, são mitigadas com adoções do programa de manutenção preditiva. Com relação a variabilidade da condução pelos motoristas, as mesmas não foram consideradas pois não interferem no padrão de cálculos.

2. Para o cálculo do custo de operação, apenas o custo de combustível foi considerado. Os custos com mão de obra diretos e indiretos para utilização da frota foram desconsiderados por política da empresa.

3. A vida máxima de utilização de um ônibus foi adotada em 12 anos, de acordo com os limites definidos pela legislação local.

4. Todos os valores de custos de manutenção em base anual foram convertidos para valores atuais de acordo com as taxas de inflação vigentes no período, de forma a permitir a comparação entre os mesmos. Para efeito de cálculo todos os custos foram atualizados para o último período de análise vigente.

5. O custo de investimento para aquisição do veículo foi considerado como o valor de compra de um veículo novo similar, no último período de análise vigente conforme informado pela empresa.

6. Para este estudo, os valores monetários foram apresentados em euros, tal procedimento foi adotado, pois este trabalho está inserido numa pesquisa maior de doutorado realizada entre universidades brasileira e espanhola. A taxa de conversão adotada coincide com data base de dezembro de 2015 ( $€ 1,00=\mathrm{R} \$ 4,33$ ). 
Com os critérios supracitados, foi possível analisar os custos de operação e manutenção, de todos os 33 ônibus durante o período de 5 anos, no qual a frota foi operada. A figura 3 indica um box-plot dos custos totais para cada período de idade dos 33 ônibus que compõem a amostra.

Figura 3 - Box-plot descritivo do custo total de operação e manutenção acumulado da amostra

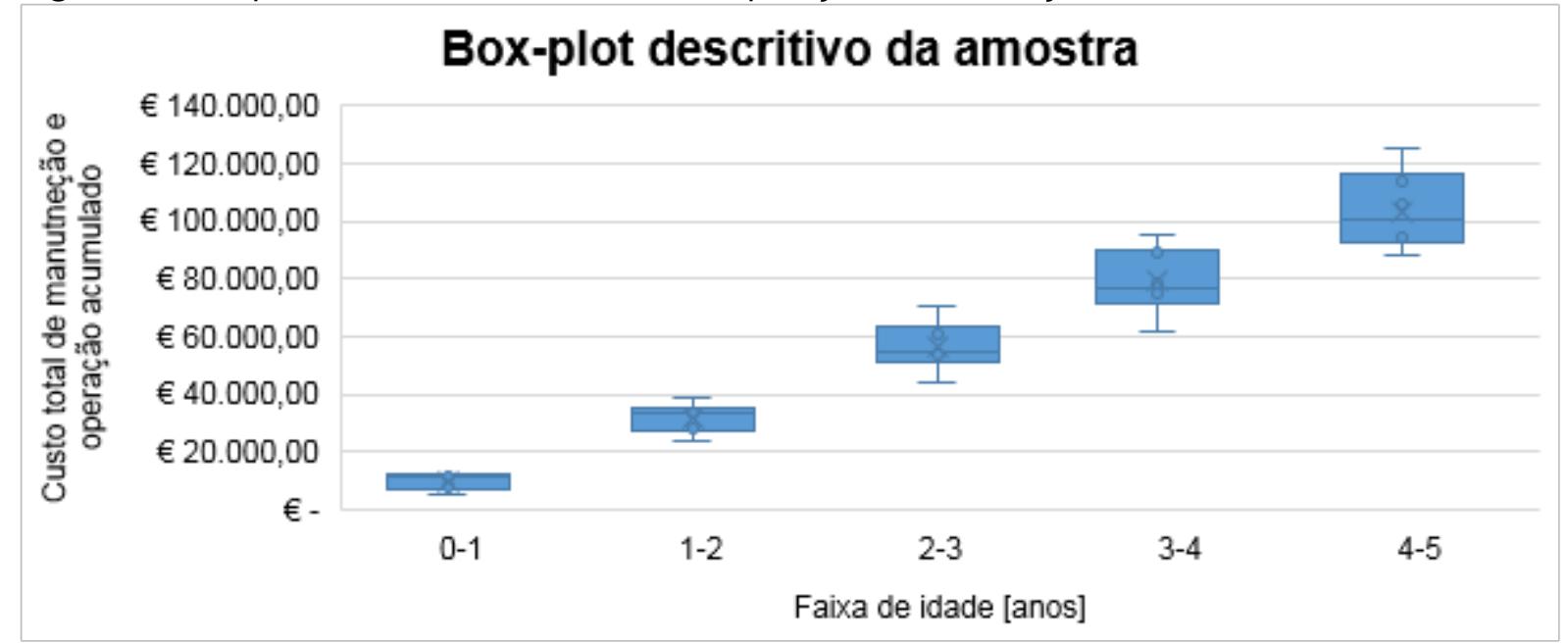

\subsection{Modelo Matemático}

Primeiramente, definiu-se uma função matemática que representava o custo total de operação e manutenção acumulado " $Y$ " até a idade " $x$ " e a quilometragem total do veículo " $y$ ", dessa forma obteve-se o descrito em (1).

$$
Y=F(x, y)
$$

Para cálculo do LCC foi necessário definir o fluxo de caixa em função da idade e da quilometragem total acumulada " $G(x, y)$ ". O fluxo de caixa foi definido como a soma dos custos totais " $Y$ " com o valor de compra do veículo " $V c$ " subtraindo o valor de revenda do veículo " $V r$ ", de acordo com o definido em (2). O valor de revenda será posteriormente explicado, porém ele foi considerado desentendendo exclusivamente da idade do veículo, sendo considerado " $\operatorname{Vr}(x)$ ".

$$
G(x, y)=Y+V c-V r=F(x, y)+V c-V r(x)
$$


Com o fluxo de caixa definido o problema passou a ser descrito como um problema de otimização ao se definir o par de ordenadas " $\left(x^{*}, y^{*}\right)$ " referente a idade ótima e a quilometragem total ótima para substituição do ônibus. Sobre a premissa de uma saída fixa, a decisão de substituição é simplesmente uma questão de o custo de operação de um veículo existente é maior que que o custo total anualizado de troca do veículo (JIN, KITE-POWELL, \& L., 2000), dessa forma esse ponto foi determinado através do cálculo do valor mínimo do custo médio anual por quilometragem ou "Average Annual Cost" de agora em diante indicado pela sigla $A A C$, de acordo com o definido em (3):

$$
H(x, y)=\frac{G(x, y)}{y}=\frac{F(x, y)+V c-\operatorname{Vr}(x)}{y}
$$

Portanto o problema foi reescrito como " $\{P 1\}$ " de acordo como mostrado em (4):

$$
\begin{aligned}
& \{P 1\}\left\{\begin{array}{c}
\text { Encontrar }\left(x^{*}, y^{*}\right) \in R^{2}, \text { de tal forma que } \\
\left(x^{*}, y^{*}\right)=\operatorname{argmin}[H(x, y)]=\arg \min \left[\frac{F(x, y)+V c-V r(x)}{y}\right]
\end{array}\right. \\
& \text { Sendo: }\left\{\begin{array}{c}
x^{*} \in\left[x_{\min }, x_{\max }\right] \\
y^{*} \in\left[y_{\min }, y_{\max }\right] \\
x_{\min }, x_{\max }, y_{\min }, y_{\max } \in R^{+}
\end{array}\right\}
\end{aligned}
$$

Com o problema apresentado em " $\{P 1\}$ " já seria possível chegar a uma a solução, porém o par de ordenadas idade e quilometragem " $(x, y)$ " permitia situações de simulação que não correspondiam à realidade de operação dos veículos, permitindo por exemplo veículos com elevado utilização em curto período de tempo e ônibus que não são quase utilizados durante toda sua vida útil fossem considerados. Para corrigir essa dificuldade operacional, foi realizado uma troca de variáveis considerando que a quilometragem média anual poderia ser assumida constante para um veículo (hipótese levantada na seção 2.1 desse trabalho), dessa forma obteve-se a quilometragem média anual " $w$ " definida em (5):

$$
y=x * w \therefore w=\frac{y}{x}
$$


Portanto, pode-se reescrever o problema " $\{P 1\}$ " em função do par de ordenadas idade e quilometragem média anual " $(x, w)$ " no problema " $\{P 2\}$ " de acordo com o defindo em (6):

$$
\begin{gathered}
\{P 2\}\left\{\begin{array}{c}
\text { Encontrar }\left(x^{*}, w^{*}\right) \in R^{2}, \text { de tal forma que } \\
\left(x^{*}, w^{*}\right)=\arg \min [H(x, w)]=\arg \min \left[\frac{F(x, w)+V c-V r(x)}{y}\right] \\
\therefore\left(x^{*}, w^{*}\right)=\arg \min \left[\frac{F(x, w)+V c-V r(x)}{x * w}\right]
\end{array}\right. \\
\text { Sendo: }\left\{\begin{array}{c}
x^{*} \in\left[x_{\min }, x_{\max }\right] \\
w^{*} \in\left[w_{\min }, w_{\max }\right] \\
x_{\min }, x_{\max }, w_{\min }, w_{\max } \in R^{+}
\end{array}\right\}
\end{gathered}
$$

Vários modelos poderiam ser adotados para representar as informações contidas no banco de dados, como por exemplo modelos polinomiais, logaritmos, até mesmo modelos lineares. No entanto, o custo de operação e manutenção total é comumente descrito por uma função exponencial devido ao seu conhecido comportamento (LAPAŠINSKAITĖ \& BOGUSLAUSKAS, 2005). Sendo assim, foi adotado um formato exponencial para a função que modelava o custo total de operação e manutenção em função da idade do ônibus e da quilometragem média anual " $F(x, w)$ " conforme definido em (7):

$$
\begin{aligned}
& F(x, w)=a * w * x * \exp (b * x) \\
& \text { Sendo } a \text { e } b \in R^{+} e \neq 0
\end{aligned}
$$

Para encontrar os valores das constantes " $a$ " e " $b$ " foi utilizado o software estatístico Minitab ${ }^{\circledR}$ que através da análise do banco de dados dos 33 ônibus utilizados e considerando o custo total de manutenção acumulados descrito em Euros e os parâmetros idade do ônibus e quilometragem média anual para cada um dos períodos anuais considerados realizou uma aproximação a função " $F(x, w)$ ", conforme defindo em (7), para tal aproximação foi utilizado a técnica dos mínimos erros quadrático. Na aproximação pelos mínimos erros quadráticos buscou-se minimizar a soma do quadrado distância entre de todos os valores observados e o valor previsto pela função definido pelas constantes " $a$ " e " $b$ ". 
A figura 4 mostra o valor da curva obtida com o resultado a técnica dos mínimos erros quadráticos tanto para a região na qual se possuía dados como para o período que consiste até a idade máxima de simulação, representado pela linha contínua central. Ressalta-se que mesmo com a base de dados cedida pela empresa limitada até um período de 5 anos de utilização, pode-se extrapolar os valores para um período superior de análise, conforme foi realizado por esse trabalho. Através de uma análise estatística foi possível determinar o intervalo de predição e o intervalo para o valor médio do custo médio anual por quilômetro com um grau de confiança de 95\%, ambos intervalos são demonstrados na figura 4 .

O intervalo de predição para o custo médio anual por quilômetro, demostrado pela linha tracejada externa, indica que com $95 \%$ de confiança é possível afirmar que um ônibus representativo da frota analisada terá um comportamento que se encaixe no intervalo indicado pelas curvas limites. O intervalo para o valor da média do custo médio anual, demonstrado pelas linhas tracejadas internas, indica que com 95\% de confiança é possível afirmar que ao se analisar uma frota de ônibus semelhante a frota analisada o seu comportamento médio estará representado por um comportamento que se adequa ao intervalo indicado pelas curvas limites.

Percebe-se que os intervalos de predição possuem um intervalo mais estreito para os anos iniciais de vida do veículo, valor que se alarga conforme o veículo é utilizado. Tal comportamento é oriundo de dois fatores principais. O primeiro fator é típico de uma análise de dados acumulados, portanto, quanto maior for a utilização do veículo, maiores são incertezas que são agregadas e consequentemente maior é o valor do intervalo de predição. O segundo fator é devido à falta de informação sobre as áreas superior a cinco anos de utilização, o que não apenas aumenta a incerteza sobre o valor predito, aumentando não apenas o intervalo de predição, como também aproximando os valores do intervalo dos valores do intervalo de predição do valor médio. 
Figura 4 - Custo médio anual por quilômetro AAC

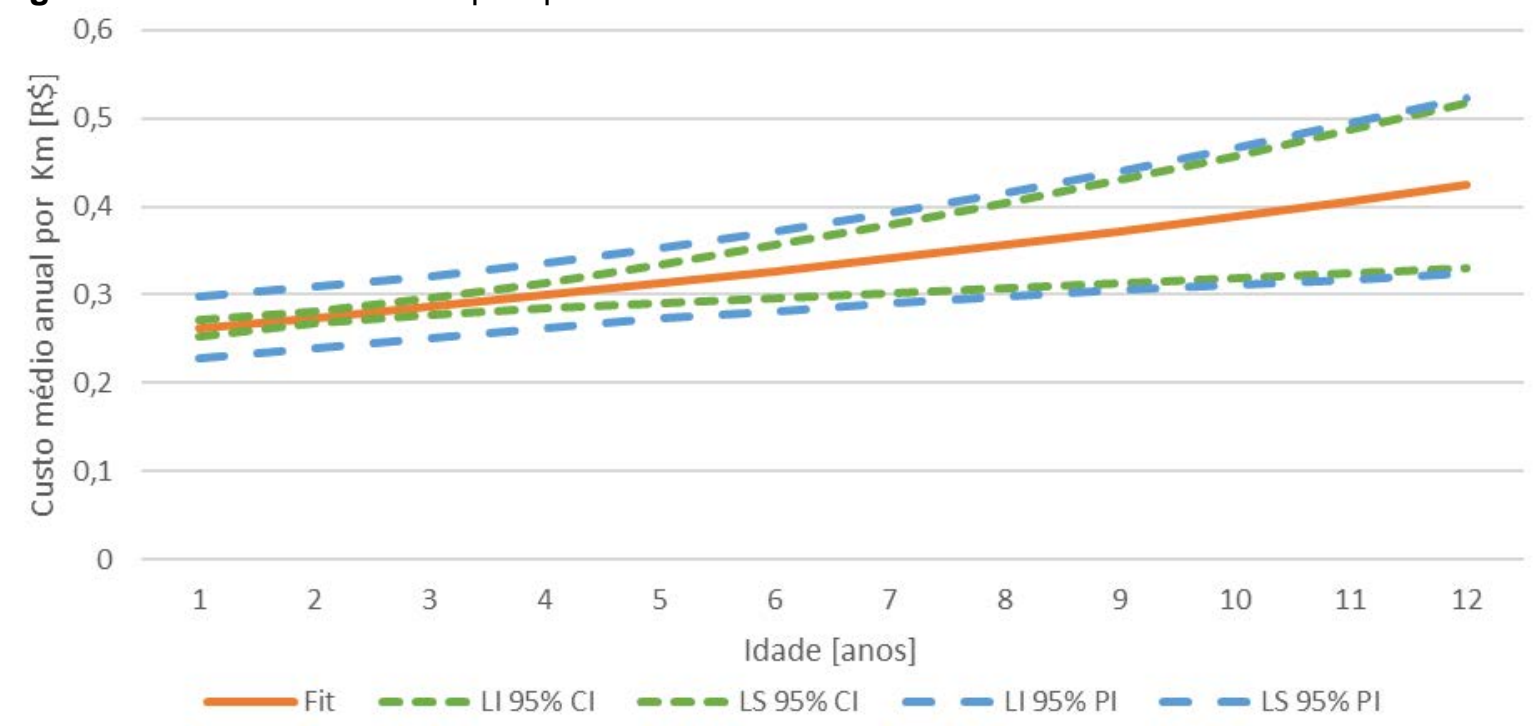

Outro indicativo estatístico muito utilizado para validar a adequação de um modelo não linear a uma base de dados é o valor da variância " $S$ " do banco de dados comparada a predição do modelo. Para os dados apresentados o software estatístico calculou a variância em 0,01618 €/Km, o que é considerado aceitável para um grau de $95 \%$ de confiança, visto que a variância é inferior a $5 \%$ do valor base de cálculo.

Para o modelo de depreciação foi considerado um modelo dependente apenas da idade do veículo, o nível de utilização foi desconsiderado pois todos os ônibus são submetidos a uma severa política de manutenção preventiva, conforme política da empresa analisada.

A formulação matemática desse modelo de depreciação, expressa pelo valor de revendo do veículo " $\operatorname{Vr}(x)$ " foi adotado conforme o modelo utilizado pela própria empresa, descrito na equação abaixo (8).

$$
V r=R+\left[\frac{V c-R}{x_{\max }} * 0,7778 *\left(x_{\max }-x\right)\right]
$$

$\mathrm{Na}$ equação (8) o valor de compra do veículo "Vc" é o mesmo descrito no início do desenvolvimento do modelo, o parâmetro " $R$ " é o valor de venda do ônibus após o final da vida útil do mesmo e o parâmetro " $x_{\max }$ " é a valor em anos da vida útil máxima do veículo. O valor de "R" é considerado como nulo quando a empresa não realiza nenhuma operação com ganho financeiro quando o veículo atinge o final 
de sua vida útil. Para esta empresa em específico o valor do parâmetro " $R$ " foi adotado como $10 \%$ do valor total de compra do veículo "Vc", premissa que será seguida por este trabalho.

\subsection{ANÁLISE DO LIFE CYCLE COST}

Para análise do $L C C$, foi utilizado a equação do fluxo de caixa conforme definido em (2), onde cada um dos elementos foi calculado separadamente, considerando o período de tempo anual até a idade máxima do veículo " $x_{\max }$ ", definida como 12 anos de acordo com a legislação local.

Os custos de operação e manutenção " $F(x, w)$ ", foram definidos de acordo com a função (7), tendo como parâmetro de utilização média anual "w" 80.620 quilômetros por ano de uso, de acordo com o explicitado na seção 2.1 deste trabalho. O modelo de depreciação utilizado foi o descrito na seção 2.2. Os valores das constantes da função custo total, conforme definido em (7), foram determinadas com o auxílio do software estatístico @Minitab.

De acordo com o modelo determinístico o momento de troca do ônibus deve ser quando o valor do custo médio anual $A A C$ atinge o seu mínimo valor, para tal este valor foi calculado de acordo com o exposto na equação (3) para todos os pontos onde o fluxo de valor foi considerado.

Os resultados dessa análise estão demonstrados na tabela abaixo e foram posteriormente discutidos. 
Tabela 1 - Resultado numérico da análise de Life Cycle Cost

\begin{tabular}{c|cccccc}
\hline \multicolumn{1}{c}{ Idade } & $\begin{array}{c}\text { Custo total de } \\
\text { O\&M } \\
\text { Acumulado }\end{array}$ & $\begin{array}{c}\text { Custo de } \\
\text { compra }\end{array}$ & $\begin{array}{c}\text { Valor de } \\
\text { revenda }\end{array}$ & $\begin{array}{c}\text { Fluxo de } \\
\text { caixa }\end{array}$ & AAC/ $\mathbf{K M}$ \\
\hline 0 & - & $€ 103.926,10$ & $€ 89.536,33$ & $€ 14.389,77$ & \\
1 & $€ 21.000,92$ & $€ 103.926,10$ & $€ 76.345,71$ & $€ 48.581,30$ & $€ 0,607$ \\
2 & $€ 43.870,11$ & $€ 103.926,10$ & $€ 64.354,24$ & $€ 83.441,97$ & $€ 0,522$ \\
3 & $€ 68.732,24$ & $€ 103.926,10$ & $€ 53.561,91$ & $€ 119.096,43$ & $€ 0,496$ \\
4 & $€ 95.719,35$ & $€ 103.926,10$ & $€ 43.968,73$ & $€ 155.676,72$ & $€ 0,486$ \\
5 & $€ 124.971,29$ & $€ 103.926,10$ & $€ 35.574,70$ & $€ 193.322,69$ & $€ 0,483$ \\
6 & $€ 156.636,15$ & $€ 103.926,10$ & $€ 28.379,82$ & $€ 232.182,43$ & $€ 0,484$ \\
7 & $€ 190.870,71$ & $€ 103.926,10$ & $€ 22.384,08$ & $€ 272.412,72$ & $€ 0,486$ \\
8 & $€ 227.840,92$ & $€ 103.926,10$ & $€ 17.587,49$ & $€ 314.179,52$ & $€ 0,491$ \\
9 & $€ 267.722,42$ & $€ 103.926,10$ & $€ 13.990,05$ & $€ 357.658,47$ & $€ 0,497$ \\
10 & $€ 310.701,06$ & $€ 103.926,10$ & $€ 11.591,76$ & $€ 403.035,40$ & $€ 0,504$ \\
11 & $€ 356.973,45$ & $€ 103.926,10$ & $€ 10.392,61$ & $€ 450.506,93$ & $€ 0,512$ \\
12 & $€ 406.747,57$ & $€ 103.926,10$ & $€ 10.392,61$ & $€ 500.281,06$ & $€ 0,521$ \\
\hline
\end{tabular}

\subsection{Análise do custo de vida de um veículo atravès da simulaçâo de Monte Carlo}

A análise de Simulação de Monte Carlo adiciona ao modelo de "Life Cycle Cost' a possibilidade de se analisar não apenas a idade ótima de substituição do ônibus " $x$ " como nos permite o par de ordenadas idade e quilometragem média anual ótimos para a troca do veículo " $\left(x^{*}, w^{*}\right)$ ". O método SMC também considera a variação nas funções custo, o que permite analisar o impacto da variação de forma abrangente para que o gerente da empresa possa tomar uma melhor decisão.

Para que o problema " $\{P 2\}$ ” pudesse ser resolvido através da Simulação de Monte Carlo é necessário que o mesmo fosse convertido para uma abordagem estocástica, através do atendimento dos dois requisitos citados abaixo (AVILA \& BECK, 2015).

1. A função de custo total " $H(x, w)$ " deve ser diferenciável e continua no intervalo " $\left(\left[x_{\min }, x_{\max }\right],\left[w_{\min }, w_{\max }\right]\right)$ " 
2. As variáveis aleatórias serão consideradas como as constantes " $a$ " e "b" da função custo de operação e manutenção " $F(x, w)$ "conforme definido em (7) e serão limitadas a um intervalo e consideradas estatisticamente independentes.

Dessa forma pode-se reescrever o problema " $\{P 2\}$ " como um modelo de resolução aleatórios definido em " $(x, w) X(\Omega, \Psi, \mathrm{P})$ ", no quais " $\Omega$ " era uma amostra do espaço de eventos, " $\Psi$ " era a álgebra dos eventos e "P" era o espaço de medição de probabilidade dos eventos. Como resultado, obteve-se o problema " $\{P 3\}$ " de acordo com o definido em (9).

$$
\{P 3\}\left\{\begin{array}{c}
\text { Encontrar }\left(x^{*}, w^{*}\right) \in\left[R^{2}, \mathrm{P}\right], \text { de tal forma que } \\
\left(x^{*}, w^{*}\right)=\arg \min [H(x, w, z)] \\
\text { onde }(x, w, z) \in R^{2} X(\Omega, \Psi, P)
\end{array}\right\}
$$

As técnicas de SMC podem ser dividas basicamente de dois tipos principais: cronológicas / sequenciais e simulações não cronológicas (SILVA, 2015). A simulação desse trabalho seguiu um modelo não cronológico através de três passos principais: definição dos parâmetros, geração das variáveis aleatórias (cenários de simulação) e resolução do problema para todos os cenários gerados. Para facilitar a análise foi criado um fluxograma de solução do método SMC apresentado na figura 5.

Para definição dos parâmetros foi utilizado três tipos de informações, as primeiras informações são relativas a características construtivas e técnicas do veículo que já foram previamente avaliadas, como por exemplo: o valor das constantes que define a função do custo total de operação e manutenção em relação a idade e a quilometragem média de um veículo, o valor de aquisição do novo veículo, o valor residual de venda de um veículo após o término de sua vida útil e a idade máxima utilização de um veículo. As segundas informações são relativas à parâmetros internos da simulação, como por exemplo os limites superiores e inferiores que define o intervalo no qual a função custo total de manutenção e operação foi avaliada, o número de simulações que será realizada e o grau de variação que foi adotado para as variáveis aleatórias. O último nível de parâmetros tratava apenas de parâmetros internos a própria simulação e não interferem no 
resultado final, onde pode-se citar alguns contadores e definições de passos para análise de redes, bem como, uma matriz de resultado em que cada linha representa um cenário de simulação e as colunas representam informações desse cenário. As duas primeiras colunas representavam os valores das variáveis aleatórias para o cenário que foi analisado, a terceira coluna indicava o valor da idade recomendada para troca, a quarta coluna indicava o valor da quilometragem média anual indicada para troca e a quinta e última coluna indicado custo médio desenvolvido por quilômetro $A A C$ de um veículo submetido aos demais parâmetros desse cenário.

Com os parâmetros definidos foi gerado uma amostra aleatória de combinações das variáveis aleatórias " $A_{i}$ ". Para este modelo foi considerado duas variáveis aleatórias com distribuição de probabilidade uniforme de forma que as mesmas serão definidas conforme descrito em (10).

$$
\begin{aligned}
& X_{i} \in\left[(1-p) * x_{i}, ;(1+p) * x_{i}\right] \\
& \text { Onde } \left.\left.x_{1}=a, x_{2}=b \text { ep } \in\right] 0 ; 1\right]
\end{aligned}
$$

Para cada amostra da combinação das variáveis aleatórias " $X_{i}$ " o problema " $\{P 3\}$ " foi resolvido, encontrando o ponto ótimo econômico de substituição do veículo representado pela sua idade de substituição indicada e quilometragem média desenvolvida. Para cada cenário foi gravado os parâmetros de simulação (variáveis aleatórias), os resultados acimas descritos (idade e quilometragem desenvolvida no momento de troca) e o resultado do custo médio por quilômetro.

Com todos os cenários resolvidos e com seus resultados registrados foi realizado uma análise de histograma dos resultados, analisando a função de distribuição de custo médio anual $A A C$ " $H(x, w)$ " e do par de ordenadas idade e quilometragem ótimos para substituição do veículo " $\left(x^{*}, w^{*}\right)$ ". 
Figura 5 - Fluxograma da Simulação de Monte Carlo

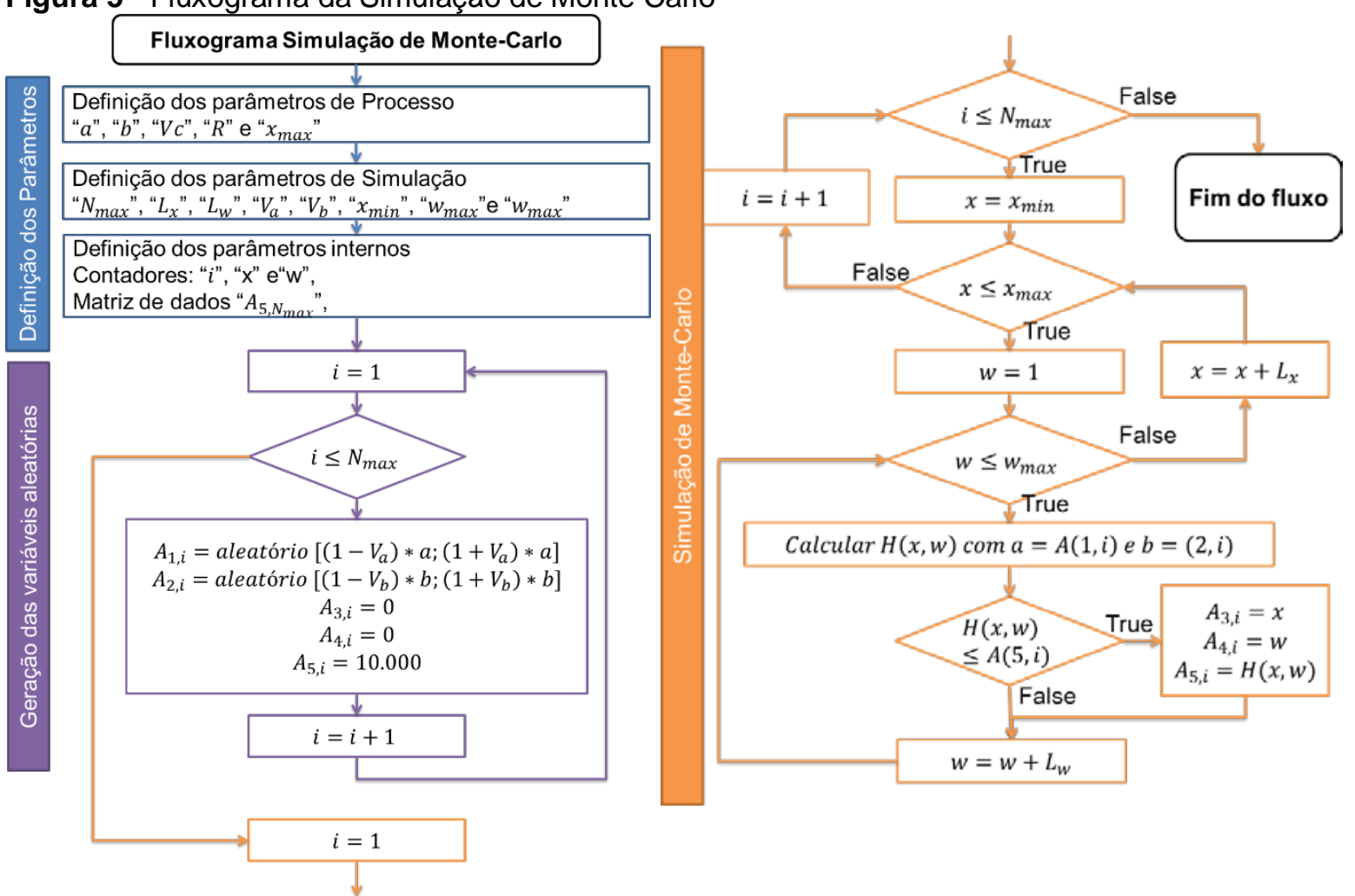

3 RESULTADOS

Com os parâmetros definidos foi realizado as duas análises descritas na seção anterior. Primeiramente, o resultado da análise convencional do "Life Cycle Cost". A primeira informação analisada foi o fluxo de caixa durante a vida do veículo, identificado na figura 6. Da mesma figura foi possível identificar o comportamento dos fatores que compõe o fluxo de caixa: o custo de investimento inicial; a política de depreciação através do custo de venda; e o custo total de operação e manutenção com seu comportamento tipicamente exponencial. Com uma análise mais profunda foi possível verificar o comportamento de cada um dos fatores, ou seja, identificouse: que o custo de investimentos inicial é constante durante todo o período de vida do veículo, o comportamento exponencial do custo acumulado total de operação e manutenção de um veículo com o aumento do período de sua vida; também se observou que o custo de revenda, indicado como depreciação, diminui com o passar da vida útil de um veículo. A soma desses fatores cria o fluxo de caixa conforme demonstrado pela linha continua pela figura 6 .

Com o fluxo de caixa definido foi possível obter a curva do custo médio por quilômetro $A A C$ de acordo com a idade do veículo, que não é nada mais que a 
divisão do fluxo de caixa pela quilometragem total do veículo de acordo com a equação 3, demonstrada na figura 7. Dessa mesma figura percebeu-se uma pequena variação de custo de no período de três a seis anos de uso, por isto, foi adicionado ao gráfico uma análise mais apurada do período de quatro a sete anos.

Dessa curva foi possível perceber que utilizando somente o modelo tradicional do "Life Cycle Cost" realizaríamos a substituição do ônibus com uma idade de 5 anos com um custo médio de $€ 0,484$ por quilômetro rodado.

Figura 6 - Life Cycle Cost de um veículo

Custo total de manutenção e operação Custo de compra

Depreciação

Fluxo de caixa

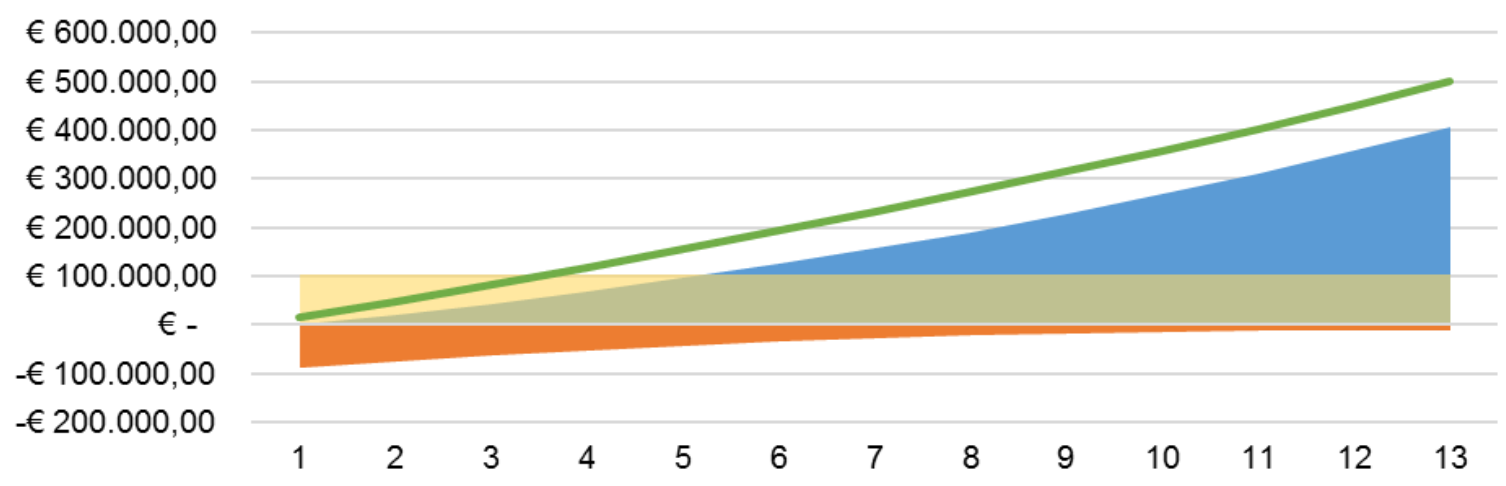

Figura 7 - Análise do custo médio anual pela idade do veículo

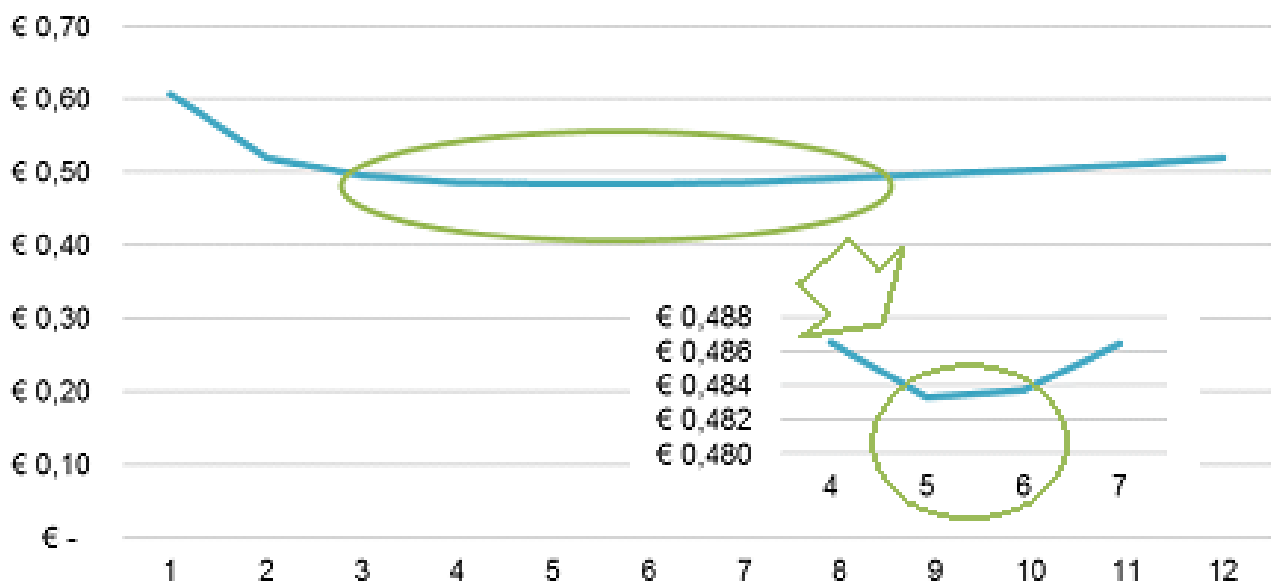

Quando se soma ao "Life Cycle Cost", a Simulação de Monte Carlo cria-se a necessidade de se analisar o resultado de toda a população e não apenas um resultado numérico simples. Permitindo ainda com este modelo combinado uma 
análise mais eficiente da quilometragem anual média de troca, sugerindo então uma política de utilização para os ônibus e não apenas uma idade de troca.

Primeiramente foi necessário observar os padrões de distribuições da idade de substituição e de quilometragem, conforme demostrados nas figuras 8 e 9. Da distribuição de idade e quilometragem anual média foi verificado que a idade ótima de troca situava-se próximo aos 4 anos de uso com utilização média anual de 100.000 quilômetros por ano. Ressalta-se que o valor de 100.00 quilômetros por anos é o valor definido como limite superior de análise, por isso o resultado sugere que quanto maior for a utilização anual menor serão os nossos resultados de custo médio anual por quilômetro, porém ressalva-se o fato de que devido à ausência de informações no banco de dados cedido pela empresa na região externa aos nossos limites, não é possível extrapolar o quanto a mais ainda é benéfico aumentar a utilização anual do veículo sem ocorrer uma perca de performance.

Figura 8 - Distribuição da Quilometragem média anual de troca ótima

\section{Quilometragem Indicada}

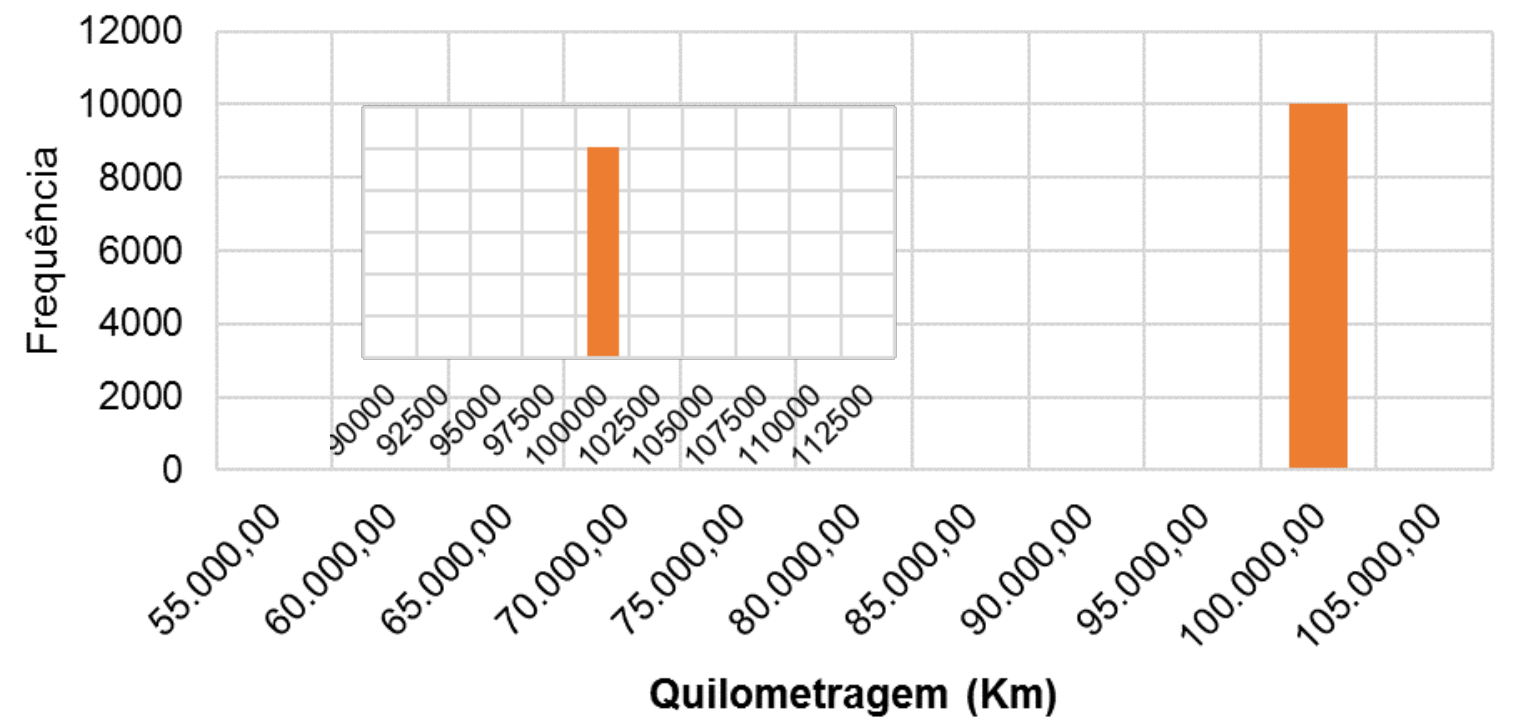

Figura 9 - Distribuição da idade ótima de troca 


\section{Idade indicada para a troca}

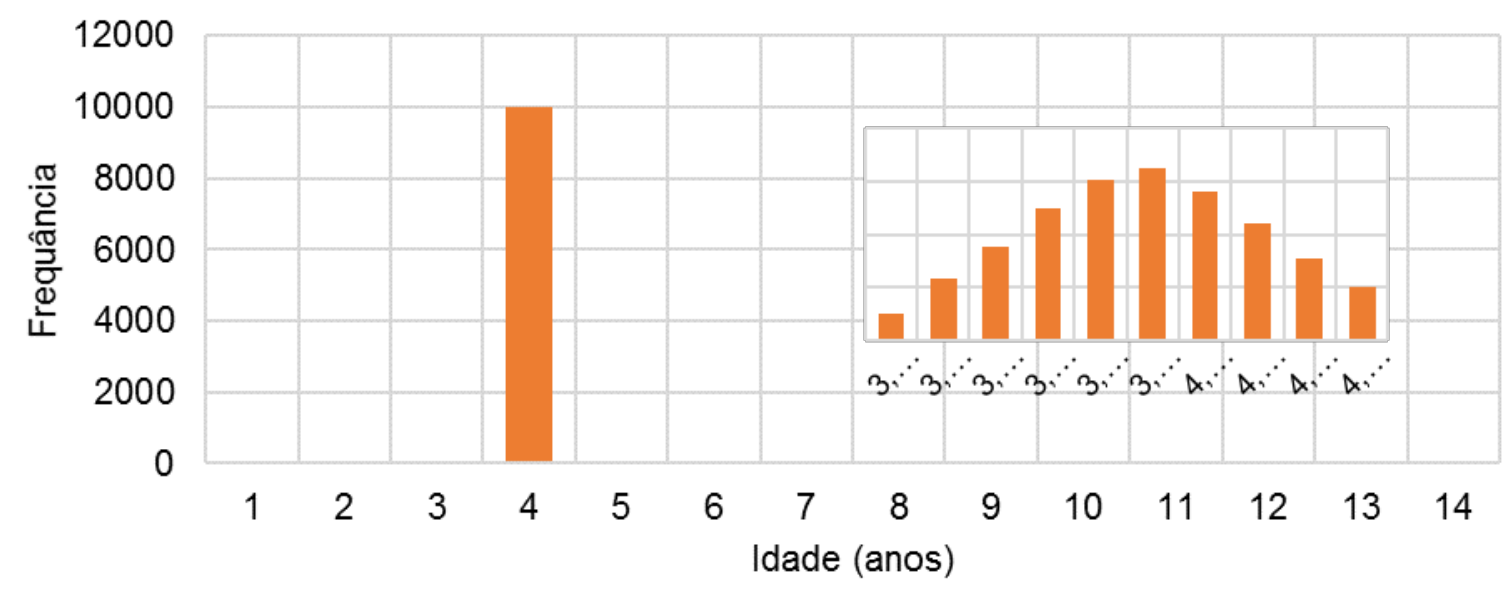

Para avaliação do custo médio por quilômetro $A A C$ não foi significativo a análise da distribuição de valores pois a mesma não teria valor comparativo para com a metodologia do "Life Cycle Cost". Dessa forma, para que se pudesse realizar uma comparação entre a metodologia do $L C C$ tradicional e o modelo proposto por esse trabalho foi realizado um mapeamento do custo médio anual por quilômetro ( $A A C)$ para um ônibus com os mesmos parâmetros que o ônibus utilizado para calcular o LCC tradicional, ou seja, o ônibus possui os mesmos parâmetros " $a$ " e " $b$ " utilizados para o cálculo da metodologia do $L C C$ tradicional. Tal mapeamento seguiu a metodologia de cálculo de rede utilizada para avaliação do $L C C$ com SMC, onde o custo médio anual por quilômetro é avaliado para todas as combinações de idade e quilometragem média anual dentro dos parâmetros definidos. Como resultado dessa análise obteve-se a figura 10 em que está representado o perfil custo médio anual por quilômetro que o veículo desenvolveria caso fosse submetido a uma utilização anual de quilometragem " $w$ " e com uma idade de troca " $x$ " através de um gráfico de curva de nível.

Figura 10 - Mapeamento do custo médio por quilômetro 


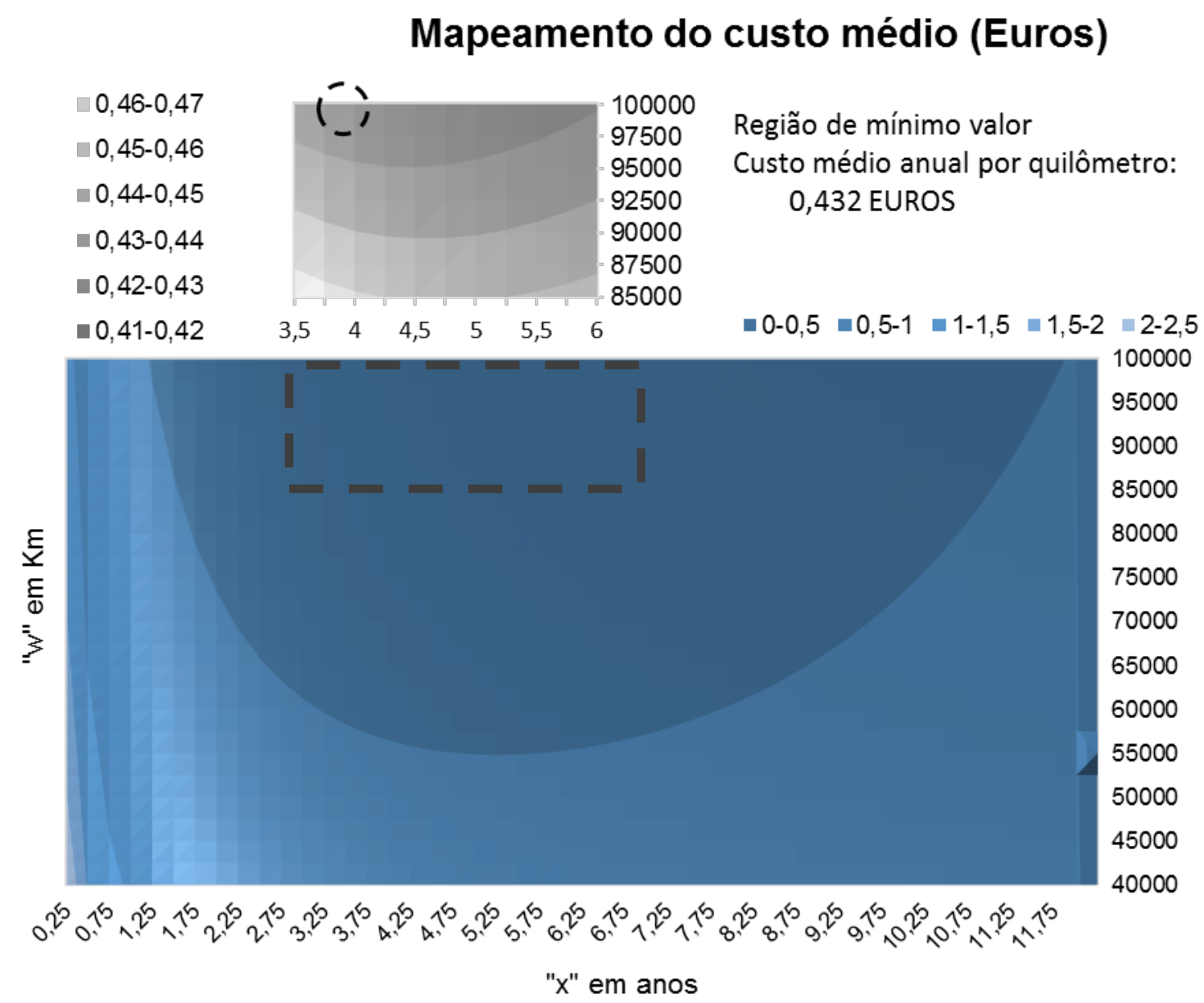

Neste gráfico de curva de nível a idade de troca está representado no eixo das abscissas enquanto que a quilometragem média anual está representada no eixo das ordenadas, o custo médio anual está representado pela cor do ponto de intersecção.

Com análise da figura, percebeu-se que existe uma a grande faixa de utilização em que o custo médio anual por quilômetro ficaria numa faixa de inferior a $€ 0,506$, porém como este trabalho tinha como intuito otimizar o ponto de troca foi necessário refinar o resultado para que se pudesse achar o par ótimo de quilometragem média anual e idade de substituição. Esse aprofundamento foi realizado através de um refinamento da rede próximo à região onde os menores valores foram encontrados, de acordo com o mostrado no gráfico de curva de nível encontrado na parte superior da figura 9. 
Da mesma, percebeu-se que ao se utilizar a sugestão do "Life Cycle Cost" com o Monte Carlo o custo médio por quilômetro atinge o valor de $€ 0,432$. Quando comparado com o resultado encontrado no modelo $L C C$ tradicional encontrou-se uma redução de $€ 0,05$ no custo anual médio por quilômetro $A A C$, mesmo que essa redução pareça pequena quando comparada no resultado por quilômetro, indica uma diminuição de aproximadamente $10 \%$ custo total de operação de uma frota de ônibus.

De acordo com as informações cedidas pela empresa $Z$ a substituição dos ônibus está programada para ocorrer quando os mesmos atingem uma idade média de 7 anos de uso com uma utilização média de 80.000 quilômetros anuais, o que indica um custo médio por quilômetro próximo de 0,506 euros como valor atual desenvolvido.

A tabela 2 abaixo sumariza os resultados obtidos para cada um dos cenários desenvolvidos de um veículo:

Tabela 2 - Comparativo entre os modelos matemáticos

\begin{tabular}{c|ccc|c}
\multicolumn{1}{c}{ Modelo } & $\begin{array}{c}\text { Idade de } \\
\text { substituição }\end{array}$ & $\begin{array}{c}\text { Quilometragem } \\
\text { anual }\end{array}$ & \multicolumn{2}{l}{ AAC/ KM } \\
\hline LCC tradicional & 5 anos & 80.000 & $€ 0,484$ & \\
LCC com Monte Carlo & 4 anos & 100.000 & $€ 0,432$ \\
Utilização da empresa & 7 anos & 80.000 & $€ 0,506$ &
\end{tabular}

Com a inclusão de uma análise estocástica ao modelo determinístico, conseguiu-se reduzir o resultado da simulação em aproximadamente 0,05 euros no custo total de operação e manutenção anual por quilômetro rodado. Tal redução representaria o impacto esperado em $10 \%$ no custo total de operação e manutenção da frota de veículos como um todo. Os resultados foram ainda mais expressivos quando comparados aos atuais desenvolvidos pela empresa, uma diminuição superior a 0,07 euros no custo total anual por quilômetro o que representaria uma redução prevista de 12\% com relação ao padrão de custo médio anual atualmente desenvolvido. Isto demonstra a eficácia do modelo combinado entre a ferramenta LCC somada a Simulação de Monte Carlo para obter resultados confiáveis em renovação de frotas (SINGH \& TIONG, 2005). 


\section{CONCLUSÃo}

Este artigo apresentou uma sistemática, combinando a ferramenta gerencial Life Cycle Cost (LCC) e o modelo matemático de Simulação de Monte Carlo (SMC), com vistas ao aprimoramento do processo de tomada de decisão no planejamento de substituição de frota de empresa de transporte urbano. Como primeira etapa, foram analisados os dados econômicos e técnicos, com o uso do método tradicional determinístico baseado em $L C C$ e em seguida, com o modelo matemático estocástico combinado de LCC e SMC. Utilizou-se a SMC no tratamento das variáveis, uma vez que, a simples adoção do método determinístico não se mostra eficiente, devido à grande amplitude da análise realizada. A análise dos custos é uma atividade gerencial complexa, que requer disciplina, determinação e precisão, porém proporciona informações seguras e essenciais à gestão das empresas.

O estudo demonstrou que a inclusão das variáveis aleatórias, no processo de determinação da idade ótima de troca, conjuntamente com a melhor taxa de utilização dos veículos, em função da quilometragem média, agrega vantagens ao processo de substituição, ao permitir uma perspectiva mais confiável dos futuros cenários, mediante a análise probabilística, dependente das variáveis econômicas e técnicas. Além disso, o conhecimento detalhado da estrutura de custos é um fator determinante e imprescindível para averiguar o uso otimizado da frota. Os aspectos econômicos e técnicos foram definidos, em concordância com a empresa estudada como, os custos associados à aquisição, depreciação, manutenção e operação dos veículos.

O estudo apresentado permite a avaliação do ciclo de vida do veículo pelos gestores e, é uma ferramenta de apoio à decisão de substuição da frota. Nas análises de dados específicos de um veículo, as diferenças encontradas entre os modelos propostos, simples e combinado, mostram-se pequenas, porém ao se considerar a totalidade da frota, resultam em significativos valores, que geram grande economia e maximização dos lucros. A tomada de decisão final deve levar em consideração, não só os critérios econômicos, mas também, os não-econômicos como, os estratégicos e administrativos das empresas.

Para trabalhos futuros, sugere-se o aumento da amostra para melhor confiabilidade dos resultados. A conclusão final deste trabalho indica que, com o 
aumento da utilização média anual dos veículos, é possível reduzir o custo médio total de operação e manutenção por quilômetro. Finalmente, espera-se que este trabalho contribua no desenvolvimento de outros estudos que envolvam a substituição de ativos em frotas.

\section{REFERÊNCIAS}

AVILA, C. R., \& BECK, A. New method for efficient Monte Carlo-Neumann solution of linear. (Elsevier, Ed.) Probabilistic Engineering Mechanics, n. 40, 2015. http://dx.doi.org/10.1016/j.probengmech.2015.02.006

COSTA, A. P., NOSSA, V., TEIXEIRA, A. M., \& TEIXEIRA, A. (2014). Otimização de custos do transporte público urbano: comprar ou vender um ônibus usado. Gestão, finanças e contabilidade, 2014, p. 73-92.

DARIO, M., SILVA, E. M., NETTO, M. S., \& PIRES, S. R. Indicadores de desempenho, práticas e custos da manutenção na gestão de pneus de uma empresa de transportes. Revista Produção Online, v. 14, n. 4, p. 1235 - 1269, 2014.

http://dx.doi.org/10.14488/1676-1901.v14i4.1450

DHILLON, B. S. Life Cycle Costing For Engineers. London - U.K.: CRC Press, 2010.

EMBLEMSVAG, J. Activity-based life-cycle costing. Managerial Auditing Journal, v. 16, n. 1, p. 17-27, 2001. http://dx.doi.org/10.1108/02686900110363447

EMBLEMSVAG, J. Life Cycle Costing: using activity-based costinf and monte-carlo to manage future costs and risks. New Jersey - USA: John Wiley, 2003.

FAN, D. W., MACHEMEHL, R., GEMAR, M., \& BROWN, L. A Stochastic Dynamic Programming Approach for the Equipment Replacement Optimization under Uncertainty. Journal of transportation systems engineering and information technology, v. 14, n. 3 , p. 76-84, 2014. http://dx.doi.org/10.1016/S1570-6672(13)60137-3

FELDENS, A. G., MÜLLER, C. J., FILOMENA, T. P., KLIEMANN Neto, F. J., CASTRO, A. S., \& ANZANELLO, M. J. Política para avaliação e substituição de forta por meio da adoção de modelo multicritério. ABCustos - Associação Brasileira de Custos, v. 5, n. 1, p. 1-27, 2010.

FENG, W., \& FIGLIOZZI, M. A. Bus fleet type and age replacement optimization: a case study utilizing king conty metro fleet data. Proceedings of the 12th conference on advanced systems for public transport, 2012.

JIN, D., KITE-POWELL, \& L., H. Optimal fleet utilization and replacement. Transportation research, E 36, p. 3-20, 2000. http://dx.doi.org/10.1016/s1366-5545(99)00021-6

LAPAŠINSKAITĖ, R., \& BOGUSLAUSKAS, V. The maintenance Cost Allocation in Product Life Cycle. Engineering Economics. v. 4, n. 44, p. 17-23, 2005. 
LEAL, L. R., \& OLIVEIRA, M. J. . Simulação aplicada ao gerenciamento de projetos: uma revisão. Revista Produção Online, v. 11, n. 2, p. 503-525, abr./jun. de 2011.

http://dx.doi.org/10.14488/1676-1901.v11i2.737

MISHRA, S., SHARMA, S., KASNABIS, S., \& MATHEW, T. V. Preserving an aging transit fleet: an optimal resource allocation perspective based on service liffe and constrained budget. Trasportation reserach Part, 47, p. 11-123, 2013.

PAMPLONA, E. O., \& SILVA, W. F. Contribuição da simulação de Monte Carlo na projeção de cenários para gestão de custos na área de laticínios. CONGRESSO INTERNACIONAL DE CUSTOS, Anais... 2005.

RITTER, F., PANDOLFO, A., BARCELLOS, L. J., RITTER, V. R., PANDOLFO, L. M., TAGLIARI, L. D., \& BARCOVI, N. E. (). Utilização do método de Monte Carlo para avaliação econômica de policultivos de jundiás. Revista Produção Online, v.14, n. 4, p. 1292-1315, out./dez de 2014. http://dx.doi.org/10.14488/1676-1901.v14i4.1590

SARAIVA JUNIOR, A. F., RODRIGUES, M. V., \& COSTA, R. P. Simulação de Monte Carlo aplicada à decisão de mix de produtos. Produto e Produção, v. 11, n. 2, p. 6-54, jun. 2010.

SILVA, J. Definition if maintenance policies in power systems using a sequential Monte Carlo. Journal of Engineering, p. 122-137, 2015.

SILVEIRA, M. C., ANZANELLO, M. J., \& ECTCHEVERRY, G. V. Programação de produção via seleção de variáveis e simulação de Monte Carlo. Revista Produção Online, v. 14, n. 2, p. 764-788, abr./jun. 2014. http://dx.doi.org/10.14488/1676-1901.v14i2.1603.

SINGH, D., \& TIONG, R. Development of Life Cycle Costing framework for highway bridges in Myamar. International Journal of Project Management, n. 23, p. 37-44, 2005. http://dx.doi.org/10.1016/j.jproman.2004.05.010

ZAMBUJAL, J. O., \& DUQUE, J. Operational asset replacement strategy: a real options approach. European Journal of Operational Research, n. 210, p. 318-325, 2011.

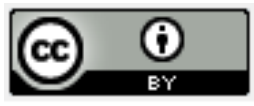

Artigo recebido em 17/11/2016 e aceito para publicação em 06/04/2017 DOI: http://dx.doi.org/10.14488/1676-1901.v17i2.2627 